\title{
Peines d'amour perdues ou le jeu des erreurs
}

\section{Sophie Chiari}

\section{(2) OpenEdition}

\section{Journals}

\section{Édition électronique}

URL : http://journals.openedition.org/shakespeare/3189

DOI : 10.4000/shakespeare.3189

ISSN : 2271-6424

Éditeur

Société Française Shakespeare

\section{Référence électronique}

Sophie Chiari, «Peines d'amour perdues ou le jeu des erreurs ", Actes des congrès de la Société française Shakespeare [En ligne], 32 | 2015, mis en ligne le 10 mars 2015, consulté le 23 juillet 2020. URL : http:// journals.openedition.org/shakespeare/3189; DOI : https://doi.org/10.4000/shakespeare.3189

Ce document a été généré automatiquement le 23 juillet 2020.

(c) SFS 


\title{
Peines d'amour perdues ou le jeu des erreurs
}

\author{
Sophie Chiari
}

1 «Sweet misprision!» (IV.iii.96) s'exclame Berowne dans l'un des nombreux apartés qui jalonnent la scène des sonnets dans Peine d'amour perdues. Si le mot «misprision » désigne en premier lieu un acte répréhensible sur le plan juridique, le terme renvoie aussi, plus communément, à la notion d'erreur ou de quiproquo ${ }^{1}$. Or, dans une pièce qui, à sa façon, se présente comme une autre « comédie des erreurs », ces deux termes y sont employés à de nombreuses reprises. En effet, si le parc de Navarre rappelle le monde d'avant la chute, le faux pas de costard, qui entretient avec Jaquenetta une relation charnelle dans les recoins d'un labyrinthe de verdure, suggère d'emblée que le ver est dans le fruit. Tout, dans la pièce, semble marqué au sceau de la faute : la langue ne cesse d'y fourcher, le savoir est enseigné par un maître incompétent, les lettres y sont tantôt mal écrites, tantôt incomprises, quand elles ne sont pas tout simplement remises au mauvais destinataire. Pire encore, les amoureux se trompent de maîtresse quand ils croient dur comme fer toucher enfin au but.

2 En somme, l'erreur y joue à cache-cache avec la vérité ${ }^{2}$ parce que l'erreur, c'est le monde du jeu, de l'illusion, de l'envers du réel, alors que la vérité, elle, dévoile le ridicule, la maladie et la mort. Elle est tout ce sur quoi Ferdinand et ses amis refusent d'ouvrir les yeux mais qu'ils finissent par découvrir par l'entremise des femmes au cours de la dernière scène. La dette à payer, désormais, c'est la diète, le long carême à venir qui succède au carpe diem. Berowne, le véritable antihéros de la pièce, porte alors bien son nom qui n'évoque pas seulement le mot « brown », la couleur de la mélancolie, mais aussi, par paronomase, l'adjectif «barren » (I.i.47, IV.ii.24, IV.iii.294) qui résonne singulièrement dans une comédie hantée par le spectre de l'infertilité.

3 Afin de comprendre quelle philosophie de l'erreur et de l'illusion Shakespeare déploie dans sa pièce au-delà de la simple force comique du malentendu et de la confusion, il s'agira, dans l'analyse qui suit, de mettre en lumière trois types d'erreurs particulièrement révélateurs. Qu'elle soit fille de l'ignorance, marque de la copia renaissante, ou trace de transgression, l'erreur shakespearienne serait-elle l'indice 
d'une nature humaine fondamentalement dévoyée, ou pourrait-elle au contraire être perçue et vécue comme une felix culpa nécessaire?

\section{1. « Poverty in wit » (V.ii.269) : l'erreur vulgaire}

4 L'écriture équivoque de la pièce fait son miel de l'erreur, et l'incompétence rhétorique des principaux personnages révèle de nombreuses maculae (ou " fautes poétiques ${ }^{3}$ ») qui guident le spectateur plus qu'elles ne l'égarent au sein de la carte du tendre shakespearienne. Le terme même d'« error » n'apparaît en effet qu'à trois reprises dans la pièce ${ }^{4}$, toutes dans le dernier acte qui figure une sorte d'épiphanie négative, où, faute d'apercevoir le bout du tunnel et d'épouser leur belle, Ferdinand et ses amis prennent enfin conscience de ce qui aura été leur aveuglement tout au long de la pièce.

$5 \mathrm{Au}$ premier abord, seules la Princesse et ses suivantes, censées être vierges (donc immaculées, ou « sans tache »), ne commettent jamais d'erreur lorsqu'elles s'expriment. Rien d'étonnant à cela puisqu'elles sont françaises. En effet, selon le lexicographe John Florio, les demoiselles de France sont les meilleures ambassadrices de la langue vernaculaire ${ }^{5}$. Car si les hommes ont le monopole de l'écriture, les femmes, elles, dominent à l'oral et savent se servir de leur langue ${ }^{6}$. Seule exception dans la comédie shakespearienne, Jaquenetta, elle qui se trompe à l'acte 4 en décrivant la princesse comme déjà reine (« one of the strange queen's lords », IV.ii.113-116). Il est difficile de savoir si pareille bévue provient de la jeune ignorante, du scribe, ou de l'étourderie de Shakespeare ${ }^{7}$. Mais si la confusion est bien faite par Jaquenetta, elle en dit long sur l'attitude de la Princesse, car elle suggère que cette dernière se comporte déjà en souveraine avant même la mort de son père.

6 Aux antipodes de cette attitude, le roi de Navarre, Ferdinand, n'est guère à la hauteur de ses prérogatives. Les vers de la scène d'ouverture sont à ce titre particulièrement éloquents, car vouloir la gloire à tout prix relève d'une erreur stratégique pour l'obtenir. Les premiers mots du roi, semblables à une épitaphe, seront d'ailleurs parodiés quelques scènes plus loin par Holofernes qui se lance dans «une épitaphe improvisée sur la mort du cerf ${ }^{8} »$. La seconde est aussi ridicule que celle du roi paraît vaine et vide de sens. À ce point de notre analyse, l'emblème de Geffrey Whitney intitulé « Ridicula ambitio » peut fournir une illustration pertinente de ce thème moral. Il évoque l'histoire d'un certain Hanno, s'ingéniant à convaincre des oiseaux qu'il a capturés qu'il est un dieu, espérant par là accéder à la gloire. Une fois libérés, les oiseaux s'envolent vers la forêt, oubliant leur mission et jusqu'au nom de leur ancien maitre, pour chanter à tue-tête leurs propres airs. Whitney en conclut que l'ambition démesurée ne peut aboutir qu'à un sentiment de honte ${ }^{9}$. Dans Peines d'amour perdues, pièce où l'on oublie les noms et où l'on est vite ridiculisé par moins ambitieux que soi, c'est aussi ce que s'attache à démontrer Shakespeare.

7 Comment, d'ailleurs, faire en sorte de perpétuer son nom alors que l'on se trompe sur celui d'autrui ? Dans la Bible, nommer quelque chose ou quelqu'un confère à celui qui nomme un pouvoir sur ce qu'il a nommé ${ }^{10}$. Dans la pièce, personne ne semble vraiment capable d'avoir la moindre autorité sur l'autre. À l'exception des jeunes femmes, chacun, à un moment ou à un autre, déforme le nom de son prochain. Camus disait que «mal nommer un objet, c'est ajouter au malheur de ce monde ${ }^{11}$ ». Or, dans l'Arcadie décomposée de Ferdinand, on écorche allègrement les noms sans penser aux conséquences néfastes que peut engendrer la désacralisation du langage. Pourtant, sous 
ses airs idylliques, le malheur de Navarre est déjà suffisamment grand pour que l'on n'en rajoute pas. Il est gravé dans le marbre : la pièce débute sur un édit obligeant de jeunes et fougueux gentilshommes à vivre en reclus et à se repaitre de savoir. «Trop bien nourri ", l'esprit des jeunes aristocrates est d'ailleurs décrit comme "grossier et $\operatorname{gras}^{12} »$ ( ( gross, gross; fat, fat », V.ii.268).

Paradoxalement, les sujets de Ferdinand sont des férus de science et d'érudition, et c'est bien là que le bât blesse. Car Peines d'amour perdues est d'abord une comédie sur l'ignorance, et même ceux qui semblent relativement épargnés par la bêtise ou la naïveté ne disposent que d'un savoir lacunaire. Aussi peut-on dire que les bévues répétées du clown sont contagieuses au sein du royaume de Navarre. Parce qu'il ne fait pas partie de la bonne société, Costard vit dans l'erreur et s'y complaît. Ce satiriste est son propre dieu et s'attribue le droit de parler et d'agir à sa guise, comme le fera d'ailleurs Jaques dans Comme il vous plaira : "I must have liberty / Withal, as large a charter as the wind, / To blow on whom I please, for so fools have » (II.vii.47-49). Tout comme Jaques s'attribue les qualités de l'Esprit Saint ${ }^{13}$, Costard se réfugie derrière le pouvoir de nommer, mal nommer, voire renommer les choses, et personne ne le délogera de l'espace à part qui est le sien.

À peine plus haut sur l'échelle sociale, les Neufs Preux brillent aussi par leurs déficiences. L'un d'entre eux, Armado, se rêve en amoureux transi, mais ne maitrise que très imparfaitement les codes de l'idéal chevaleresque ${ }^{14}$. Aussi le torchon de Jaquenetta ("dishclout», V.ii.689) qu'il serre sur son cœur peut-il être interprété comme une première version, comique et grotesque, du mouchoir de Desdémone. Ce torchon est, lui aussi, une synecdoque, particulièrement déplaisante même si elle se veut comique, puisqu'elle assimile sa propriétaire à une souillon ${ }^{15}$. En d'autres termes, tout en pensant rendre hommage à sa dulcinée, l'Espagnol ne fait que la dénigrer un peu plus en soulignant sa basse extraction sociale.

Quant aux Neuf Preux pris dans leur ensemble, ils sont tout aussi ineptes. Même le maître d'école Holofernes se trompe dans ses choix scéniques destinés à éblouir son royal auditoire ${ }^{16}$ et, lorsqu'il s'exprime, il produit une sorte de monstre rhétorique ${ }^{17}$ qui ne peut qu'induire en erreur ses élèves et, plus largement, ses interlocuteurs.

\section{2. "The letter is too long by half a mile » (V.ii.54) : de l'excès à l'erreur}

11 Holofernes est donc celui qui, dans la pièce, échoue à éduquer et à convaincre parce sa rhétorique est celle de l'excès, y compris sur le plan phonétique. Aussi insiste-t-il lourdement sur l'importance qu'il y a, à ses yeux, de bien prononcer chaque lettre d'un mot :

I abhor such fanatical phantasimes, such insociable and point-devise companions, such rackers of orthography, as to speak 'dout' sine ' $b$ ', when he should say 'doubt'; 'det' when he should pronounce 'debt'-d, e, b, t, not d, e, t. He clepeth a claf, 'cauf' half, 'hauf'; neighbour vocatur 'nebour'; 'neigh' abbreviated 'ne.' This is abhominable, which he would call 'abominable'. It insinuateth me of insanie. (V.i.15-21)

12 Sans doute le maître d'école applique-t-il ici avec un peu trop de zèle les règles énoncées dans d'innombrables traités de phonétique en vogue à l'époque, comme celui 
de G. De la Mothe, The French alphabeth (1592) ${ }^{18}$. De ce La Mothe, on sait seulement qu'il était un pédagogue français réfugié en Angleterre ${ }^{19}$ (son nom a peut-être inspiré à Shakespeare celui de Moth). Son ouvrage, qui bénéficia d'une certaine renommée aux $\mathrm{XVI}^{\mathrm{e}}$ et XVII ${ }^{\mathrm{e}}$ siècles, prodigue des conseils comme ceux que l'on rencontre à la page 8 de son traité bilingue : "pronounce distinctly. Softly, / Make no haste, open your mouth. / That is very well: that is well said ». Après avoir disserté sur la nécessité de s'exprimer dans une langue distinctement articulée, le pédagogue français entreprend d'expliquer à son lecteur qu'il faut bien prononcer toutes les voyelles, mais que de nombreuses consonnes doivent en revanche rester muettes ${ }^{20}$. Or, on l'aura compris, Holofernes, prend précisément les préceptes de De la Mothe à rebours, tout comme il récite l'alphabet à l'envers à la demande («What is 'a,b' spelled backward [...]», V.i. 40-41).

13 Abécédaires à l'envers et règles phonétiques inversées font ici partie d'un univers rabelaisien ${ }^{21}$ où copia et nonsense le disputent à la felix culpa dont le but final ne vise à rien d'autre qu'à faire rire et à libérer le royaume de Navarre de ses carcans et de règlements si sévères qu'ils confinent à l'absurde. Le pédagogue a en outre le tort de recourir à l'empilement de noms ou d'épithètes - trope que Puttenham nomme « congeries » ou " heaping figure ${ }^{22}$ ». Il se fourvoie donc parce qu'il en fait trop. De fait, tout dans la comédie grouille, dépasse et déborde, ce qui est sans doute le comble du paradoxe pour une pièce par ailleurs marquée au sceau de l'inachèvement.

La répétition excessive et fautive participe ainsi d'une stratégie d'aveuglement collectif : on parle pour ne pas penser. L'utilisation abusive de «l'envoy » est un bon exemple de ce procédé somme toute assez pathétique. Le mot, d'origine française, n'apparaît pas moins de seize fois dans l'acte III, scène i. Or, Armado se trompe dès le départ (3.1.60) en maintenant l'article français devant "envoy» alors que ce terme avait déjà été absorbé par la langue anglaise et qu'il devrait donc dire, selon toute logique, "the envoy». La définition qu'il en donne est certes intéressante, mais incomplète: "[...] it is an epilogue or discourse to make plain / Some obscure precedence that hath tofore been sain » (III.i.69-70).

En 1611, Randle Cotgrave donnera quant à lui une définition beaucoup plus précise de ce terme : « A message, or sending; also, th'Enuoy, or conclusion of a Ballet, or Sonnet; in a short stanzo by itselfe, and seruing, oftentimes, as a dedication of the whole ${ }^{23} » . \mathrm{Ce}$ qui frappe dans cette explication, c'est la relation étroite que semble entretenir le terme d' " envoy » avec la danse et la poésie - une relation qui fait sens dans la pièce, mais qu'Armado, pourtant en charge des divertissements de la cour, semble ignorer. Il est à ce titre particulièrement ironique de constater que l'Espagnol mentionne sans le savoir la conclusion d'un ballet dans une comédie où jamais une danse ne connaît le moindre début d'exécution. En somme, la langue sert ici à pallier les déficiences du réel. En outre, en associant l'idée d'un commencement ("Come, thy l'envoy begin ", III.i.60) à celle d'une conclusion, Armado brouille les pistes. Loin de mettre fin à l'histoire de Costard, il lui donne l'occasion de prendre des proportions démesurées ${ }^{24}$. Amoureux du signifiant aux dépens du signifié, le clown décompose en effet les syllabes $\mathrm{du}$ discours qu'on lui sert pour réinterpréter chaque réplique à sa guise. Aussi n'entend-il que la seconde syllabe du mot "envoy», c'est à dire "oie», terme alors synonyme de "prostituée", avant de donner libre cours à sa logorrhée. Trace du désordre babélien, le mélange d'anglais et de français qui caractérise certaines des 
scènes de la pièce rappelle aussi à qui veut l'entendre que, dans la tradition biblique, "la diversité linguistique n'est nécessaire que pour s'adapter au monde de la faute ${ }^{25}$ ".

Les lettres fautives qui circulent dans le royaume de Navarre posent précisément le problème de la démesure et du trop-plein que personne ne parvient à réguler. Maria se plaint de la missive, excessivement longue, que lui adresse Longueville, alors même que ce dernier vient de lui offrir des perles. Les quatre jeunes gens écrivent des poèmes qui ne font pas mouche tout simplement parce qu'ils se trompent dans leur manière d'écrire. Ils en font trop, et c'est ce que George Puttenham désigne par le terme de «periergia » qu'il glose par « over-labor » :

Another point of Surplusage lieth not so much in superfluity of you words, as of your travail to describe the matter which ye take in hand, and that ye over-labor yourself in your business. We call it Over-Labor, jump with the original, or rather, the Curious, for his overmuch curiosity and study to show himself fine in a light matter ${ }^{26}$.

Le sonnet du roi (IV.iii.20-36) est un exemple significatif de "periergia», puisque le souverain met tant d'ardeur à sa tâche qu'il écrit à sa dulcinée un poème d'amour pétrarquiste de seize vers là où, habituellement, un sonnet en compte quatorze. Cette pseudo-perfection constitue le défaut même du sonnet, et loin d'être le dangereux supplément derridien, à la fois addition et substitution ${ }^{27}$, le distique final retranche et efface tout ce que les vers précédents auraient pu avoir de touchant.

Selon Derrida, «[1]e signe est toujours le supplément de la chose même ${ }^{28}$ ». Alors que la Princesse et ses suivantes, masquées, viennent d'échanger leurs atours pour mieux berner leurs prétendants, Berowne comprend tout à coup l'erreur de jugement qu'il a commise et qu'il explicite au profit de ses trois amis : " and then we, / Following the signs, wooed but the sign of she » (V.ii.468-469). De prime abord, cette fois, l'erreur des jeunes gens semble pardonnable. Car, en modifiant profondément le concept de supplément et en désolidarisant le signe de la chose, les Françaises ont fait du signe un avatar trompeur, et lui ont retiré sa fonction. Néanmoins, les dames n'ont pas échangé leurs habits : elles n'ont fait qu'échanger les faveurs offertes par les soupirants. On ne peut donc s'empêcher d'être surpris par la naïveté des jeunes hommes, tant les déguisements des dames sont minimaux. En véritables Narcisses, ils ne se sont fiés qu'aux signes, aux objets envoyés par eux-mêmes, oubliant au passage les traits physiques de leurs détentrices. La Princesse, on le sait, est la plus grande des quatre (IV.i.45), Rosaline se distingue par sa peau sombre, Katharine a des cheveux couleur d'ambre (IV.iii.79) : dès lors, comment se tromper sur l'identité des Françaises, même masquées? Inversement, les jeunes femmes reconnaissent immédiatement leurs soupirants alors que ces derniers sont déguisés de la tête au pied et portent un costume moscovite ${ }^{29}$.

Par certains aspects, ces très lucides demoiselles ressemblent fort aux dames courtisées et raisonnables dépeintes dans les manuels de l'époque, comme celui d'Angel Day, The English Secretorie $(1586)^{30}$, où l'art de séduire l'aimée par voie épistolaire se trouve dûment expliqué. Toutefois, les Françaises, pour se conformer parfaitement aux directives d'Angel Day, devraient normalement réagir par écrit aux poèmes qui leur sont adressés. Une dame courtisée se doit en effet de répondre à l'auteur de la lettre. Or, refusant de rédiger le moindre billet doux, les Françaises se gardent ainsi de céder à la copia rhétorique de Navarre pour mieux renvoyer leurs soupirants dans les cordes. 
De toute manière, ces amoureux-là manquent singulièrement de sagesse et de lucidité avant même d'écrire la moindre lettre. Ils ont d'emblée choisi la mauvaise voie pour accéder au mariage puisqu'ils croient être capables de se conformer aux idéaux monastiques des "Inns of court ", ces écoles de droit londoniennes où toute présence féminine était strictement interdite, même en cuisine. Car comme l'atteste un règlement de Gray's Inn datant de 1581 aucune femme de moins de quarante ans ne pouvait y être employée en qualité de cuisinière ou de blanchisseuse ${ }^{31}$. Signer le pacte de Ferdinand, telle est donc l'erreur suprême commise par des jeunes gens pleins de fougue dans ce qui ressemble à une version grotesque du pacte faustien au cours duquel le personnage-titre, sceptique aiguillonné par la sensualité et les plaisirs du monde, lègue son âme à Lucifer ${ }^{32}$. La réticence de Berowne rappelle ainsi, par certains aspects, la réticence de Faust lui-même, et Berowne, tout comme Faust, finira par se laisser convaincre. Mais à l'inverse du protagoniste tragique de Marlowe, il aura le choix du parjure.

\section{3. « Most maculate thoughts » (I.ii.76) : l'erreur transgressive}

21 À bien y réfléchir, le parjure n'est pas l'apanage des lords. Songeons au document dont la Princesse rappelle l'existence à Ferdinand pour lui prouver que l'Aquitaine doit revenir à la France. Le document en question, ou « acquittance» (II.i.157), est promis pour le lendemain... Or, jamais on ne verra la moindre preuve juridique entre les mains de la princesse. On est là, une nouvelle fois, dans le principe de désir, où l'attente se substitue à la réalité et la frustration au plaisir.

La Princesse n'est donc pas toute blanche. Qui plus est, bien que prétendument vierges, ces demoiselles ne manquent jamais l'occasion de faire un sous-entendu grivois, et nombreux sont les critiques qui ont souligné le caractère éminemment transgressif de la langue de cette comédie où obscénité et scatologie font bon ménage. Les objets poétiques traditionnels se voient détournés à des fins licencieuses. L'œil, au cœur de la poésie pétrarquiste, prend ainsi dans Peines d'amour perdues des connotations ouvertement érotiques. En témoigne la scène de la rencontre des "lords» et des "ladies» au sein du royaume de Navarre, où l'antanaclase audacieuse sur les homophones « eye » et « ay » semble parfaitement délibérée :

ROSALINE. My physic says 'ay'.

BEROWNE. Will you prick't with your eye?

(II.i.184-85)

De toute évidence, Berowne feint de mal comprendre ce que lui dit son interlocutrice de sorte que le sentiment amoureux suscité par cette dernière prend ici un tour purement charnel. Pour Shakespeare, comme pour Bataille ${ }^{33}$, l'œil accomplit en fin de compte la fonction érotique de la vision.

Les personnages se trompent tous plus ou moins à dessein dans l'utilisation d'un mot ou d'une expression afin de pouvoir dissimuler des propos osés derrière la façade d'une naïveté de bon aloi. Souvent commentée, la scène de la chasse est un modèle de transgression langagière ${ }^{34}$. Mais attachons-nous plutôt à celui par qui la transgression arrive : Costard, l'avatar du " trickster » élisabéthain dans la pièce. Alors qu'il est sur le point d'aller en prison, Moth s'exclame: «Come, you transgressing slave, away!» 
(1.2.126). Or, dans l'Angleterre élisabéthaine, la transgression n'est rien d'autre qu'une erreur au sens large du terme comme l'explique Randle Cotgrave dans son dictionnaire Français / Anglais :

Erreur: $\mathrm{f}$.

An error, ouersight, ouerslip, mistaking; an ignorance, or false opinion; a wandering, or straying out of the right way; also, a misse, fault, offence, transgression; $[. . .]^{35}$.

Costard étant la transgression faite homme, on comprend difficilement la raison pour laquelle Berowne (III.i.147) et Armado (III.i.114) le choisissent comme messager, d'autant que le clown ne dissimule pas sa malice. On a souvent dit que costard commettait des erreurs du fait de son incompétence, mais on ne s'est pas suffisamment demandé s'il ne remettait pas sciemment le courrier du cœur qu'on lui confie à la mauvaise personne ${ }^{36}$. Car c'est un excellent moyen, pour lui de prendre l'ascendant sur son rival Armado tout en achevant de le ridiculiser aux yeux de la cour. D'ailleurs, sa première erreur de distribution devrait l'amener à rectifier le tir et à donner la seconde missive à la bonne destinataire, mais il n'en fait rien. Un constat s'impose donc ici : transgresser les ordres donnés par les auteurs des lettres, c'est pour Costard une manière d'acquérir le pouvoir que son statut social ne lui accorde pas d'emblée.

L'erreur, dans Peines d'amour perdues, se décline par conséquent sous toutes ses formes, et si elle est parfois commise par ignorance ou par excès de zèle, elle est aussi délibérée et ouvertement transgressive. Rien d'étonnant à cela dans une pièce placée sous le signe de Cupidon, l'enfant espiègle incarné ici par le malicieux Moth. Qualifié de «tender Juvenall» (I.ii.10) dans la pièce, le page est par ailleurs créé à l'image de Thomas Nashe $^{37}$, l'Arétin anglais jamais à court d'écrits licencieux et pornographiques ${ }^{38}$. Les mots du bien-nommé Moth / Cupidon sont ainsi autant de flèches qu'il lance avec justesse en plein cœur de ses cibles. Dans sa Mythologie initialement publiée à Venise en 1567, Natale Conti attribue des pouvoirs phénoménaux au dieu de l'amour : «[...] ce qui estoit laid \& difforme, il le faisoit trouver beau \& honneste ${ }^{39}$ ». Le très catholique Armado décide ainsi d'épouser Jaquenetta sans bien savoir si elle est « honneste». Les jeunes nobles sont tout aussi aveuglés par l'amour. Esthète narcissique, Berowne s'éprend d'une jeune femme à la peau sombre alors que les canons de beauté de l'époque vantent la blondeur et le teint incarnat. Katharine peut bien avoir le visage vérolé (c'est-à-dire « full of O's », V.ii.45), Dumaine tombe fou amoureux d'elle. Maria est issue de la lignée douteuse des Falconbridge, que Shakespeare exploite dans Le roi Jean, mais Longaville n'y voit que du feu. Quant à la Princesse, bien que sa visite soit explicitement motivée par la diplomatie et l'appât du gain, elle plaît à Ferdinand sans même avoir besoin de le séduire. En somme, Cupidon fait surgir le sentiment amoureux de situations incongrues, à mille lieues des sages et ennuyeux principes couchés noir sur blanc dans les pages des manuels de conduite en vogue à l'époque. Dans son traité de mythologie, Conti précise d'ailleurs qu'Amour aurait été engendré par le chaos: «Il semble qu'Hesiode en sa Theogonie vueille dire qu'Amour ou Cupidon soit issu de cette antique matiere informe, lourde, obscure, pesante \& immobile, qu'on a nommée Chaos $[. . .]^{40}$ ». Cette "matière informe, lourde, obscure » et "pesante ", c'est bien celle qui domine dans le royaume du roi Ferdinand - et qui est faite de l'étoffe même des songes.

L'étoffe de la Navarre shakespearienne est en effet tissée de non-dits, de fantasmes et d'erreurs. En somme, il y fait nuit en plein jour. Cette forme d'aveuglement est propice 
à la folie comme au renversement des conventions. Aussi les erreurs de registre des Françaises, clairement intentionnelles, sont-elles destinées à choquer. Même Costard, pourtant prompt à la plaisanterie grivoise, semble abasourdi par leur vulgarité : « $\mathrm{O}$ my troth most sweete jests, most inconie vulgar wit » (IV.i.135), s'exclame-t-il à l'issue de la scène de la chasse ${ }^{41}$. Ici, c'est le terme argotique $d^{\prime}$ ' inconie » qui retient l'attention du spectateur. L'OED note une seule occurrence précédant celle de Peine d'amour perdues, qui se trouve dans The Jew of Malta, de Christopher Marlowe où ce dernier joue sur la proximité phonétique d'«incony» et de "coney ${ }^{42}$ ». Il en va de même pour Shakespeare, qui utilise cet adjectif pour ses connotations obscènes. Ainsi, l'« inconie wit » de Maria ne désigne pas seulement son esprit subtil, mais aussi son «con » qui se trouve donc ici doublement désigné ${ }^{43}$. Voilà qui surprend d'autant plus le clown que la demoiselle, dont le prénom renvoie sans doute par erreur (ou par ironie) à la pureté virginale, se montre ouvertement grivoise.

Néanmoins, si Maria commet l'erreur de choquer un homme qui ne se privera sûrement pas de répéter ce qu'il a entendu, elle prend des risques calculés. Comme le prouve un dénouement en forme de pirouette, la belle n'a nulle envie de se marier, de sorte que repousser d'hypothétiques prétendants fait partie d'une habile stratégie de défense. Plus généralement, les quatre jeunes femmes choisissent toutes, à un moment ou à un autre, de transgresser les codes de la bienséance qui menace de les étouffer. Mais à force de détourner ces codes, elles ne sont plus capables de les lire correctement. Aussi se trompent-elles lourdement sur les intentions de leurs prétendants, malgré les signaux envoyés par ces derniers ${ }^{44}$.

28 Les billets doux de Navarre et de ses amis ont été pris pour de vulgaires farces. À cela, deux explications: soit les jeunes femmes ont mal interprété à dessein les lettres qui leur étaient destinées pour échapper au mariage (mais alors, éconduire directement les prétendants eût été chose aisée), soit elles n'ont pas su lire entre les lignes maladroites de leurs amants putatifs. Admettons. Les jeunes Françaises ne seraient donc guère plus lucides que leurs prétendants. La future reine de France pourra-t-elle, dans ces conditions, comprendre son peuple et servir les intérêts de son pays? On peut en douter.

29 Si la question du pouvoir et de sa fragilité taraude tant Shakespeare, c'est parce que l'autorité humaine est généralement défaillante. Provocation ou pure comédie ? Dans la pièce, les agents du pouvoir sont essentiellement catholiques puisque l'autorité revient à la Princesse et à ses amies, qui sont françaises. Au-delà de ces ambassadrices de charme, celui qui incarne le mieux le catholicisme fautif moqué par les Anglais, c'est le «magnifique Armado» (I.i.188) ${ }^{45}$, personnage fantasque venu d'Espagne, contrée alors dirigée par un membre de la dynastie des Habsbourg, le roi Philippe II (1527-1598), qui soutenait en France les forces catholiques de la Ligue qui combattaient Henri IV. Apparemment bien inoffensif, l'Espagnol raffole d'autant plus des bons mots de son page que lui-même en est incapable. Aussi s'extasie-t-il lorsque son serviteur entreprend d'enseigner l'alphabet à Holofernes pour mieux ridiculiser ce dernier :

мотн. The last of the five vowels, if 'you' repeat them; or the fifth, if ' $I$ '.

HOLOFERNES. I will repeat them: a, e, I -

мотн. The sheep. The other two concludes it: o, u.

ARMADO. Now by the salt wave of the Mediterraneum, a sweet touch, a quick

venue of wit!

(V.i.45-50) 
part, on comprend ici que le matamore se réjouit de la plaisanterie de Moth qui vient de faire dire à Holofernes qu'il est un mouton ( I I The sheep »). De l'autre, s'il complimente la vigueur intellectuelle de son page, c'est peut-être aussi parce qu'il identifie immédiatement le message subliminal divulgué par petit serviteur qui vient de réciter les voyelles dans l'ordre : a, e, i, o, u. À l'époque de Shakespeare en effet, AEIOU est l'acrostiche latin de la devise des Habsbourg (« Austriae Est Imperare Orbi Universo $\left.^{46} »\right)$, éminente puissance catholique qui régnait alors sur une grande partie de l'Europe.

On voit que, derrière l'incompréhension, les quiproquos, les plaisanteries et les jeux de mots grivois, la menace de l'Autre est bel et bien présente dans cette comédie grinçante, où quelques voyelles a priori insignifiantes servent à signaler la montée en puissance d'une force politique et religieuse que l'Angleterre protestante n'aura dès lors de cesse de combattre au cours des décennies suivantes. S'il est évidemment hors de question de trouver un sens à tous les jeux de lettres shakespeariens, l'interprétation est ici si tentante que la peur de l'erreur ne saurait véritablement la mettre à mal...

\section{Envoy : éloge de la delectatio morosa}

Si Peines d'amour perdues semble éprouver quelque jubilation à ne tenir aucun compte $\mathrm{du}$ traditionnel adage latin qui trouve diabolique de persévérer dans l'erreur, la comédie fait en revanche flèche de toute infraction en se jouant des (in)certitudes du langage. Force est de constater qu'au sein du royaume de Ferdinand, il est impossible de prendre pour argent comptant ce que disent les personnages : leurs paroles se retournent aisément, et leur envers n'est souvent guère reluisant. Shakespeare nous invite ainsi à pénétrer dans un royaume où le scepticisme est roi et s'articule à une foi vacillante car, comme l'erreur, le doute libère.

Curieusement, alors que le dénouement approche, l'erreur persiste dans les actes et les décisions des personnages. Alors qu'ils sont les premiers à cultiver l'erreur à leur insu, la jouissance qu'ils éprouvent à la pourfendre montre bien la fascination qu'elle exerce sur le monde. Jamais on ne se lasse de repérer l'erreur tant la delectatio morosa nous pousse à jouir de la faute ${ }^{47}$. Qui s'intéresserait à un personnage parfait ou à une œuvre dépourvue de faiblesses? L'erreur est sans aucun doute la meilleure manière de devenir célèbre, et les gentilshommes l'apprennent rapidement à leurs dépens.

Il faut dire que, dans le royaume de Navarre, se tromper c'est vivre. Autre façon de dire que la perfection n'est pas de ce monde et si, en anglais, l'erreur est souvent signalée par le préfixe «miss", une ultime pirouette nous permettrait d'affirmer que, d'une certaine manière, Peines d'amour perdues est bel et bien une comédie mettant les « mis(s) » à l'honneur. Néanmoins, je terminerai mon " envoy » sur une réflexion plus personnelle. Et si la plus grave erreur des jeunes hommes n'était pas, finalement, de croire que les demoiselles de France veulent vraiment être courtisées et qu'eux-mêmes sont réellement capables de s'offrir à elles ? Bien avant Lacan, Shakespeare semble avoir compris que l'amour, c'est « donner ce qu'on n'a pas à quelqu'un qui n'en veut $\operatorname{pas}^{48}$ ». Immatures, les gentilshommes semblent ne chérir que l'idée même de l'amour. Quant à la Princesse, jeune cynique en herbe, elle possède des raisons purement diplomatiques de vouloir plaire au roi de Navarre. Reste donc, pour Ferdinand et ses

Actes des congrès de la Société française Shakespeare, 32 | 2015 
amis, ce qu'on nomme en anglais le "male-bonding », une solidarité et une affection toute masculine qui, loin des femmes savantes, serait vraiment à même de promouvoir une philosophie de l'errance et du tâtonnement.

\section{BIBLIOGRAPHIE}

Bataille, Georges, Histoire de l'œil (1928), Paris, Gallimard, 1993.

Campbell, Oscar J., « Love's Labour's Lost Restudied (1925) » in Love's Labour's Lost. Critical Essays, éd. Felicia Hardison Londré, New York, Routledge, 2001, p. 83-116.

Camus, Albert, Euvres complètes, tome I, éd. Jacqueline Lévi-Valensi, Gallimard, coll. « La

Pléiade », 2006.

Cicéron, De Oratore, Livre III, 44-46, trad. H Rackham, Cambridge, Loeb Classical Library, 1982.

Compagnon, Antoine, La seconde main ou le travail de la citation, Paris, Seuil, 1979.

Conti, Natale, Mythologie c'est à dire, Explication des Fables contenant les genealogies, Lyon, 1600 .

Cotgrave, Randle, A Dictionarie of the French and English Tongues (Londres, 1611), facs., éd. William S. Woods, Columbia, The University of South Carolina Press, 1950.

Crystal, David, 'Think on my Words'. Exploring Shakespeare's Language, Cambridge, CUP, 2008.

Curtis, Benjamin, The Habsburgs: The History of a Dynasty, London, Bloomsbury, 2013.

Day, Angel, The English Secretorie, Londres, imprimé par Robert Waldegrave, 1586, STC nº 6401.

De la Mothe, G., The French alphabeth, Londres, 1603, STC nº 6547.

Derrida, Jacques, De la grammatologie, II, 2, Paris, éditions de Minuit, 1967.

Fleming, Juliet, « Dictionary English and the Female Tongue » in Privileging Gender in Early Modern England, éd. Jean R. Brink, Sixteenth Century Essays and Studies, vol. 23, Kirksville, MO: Sixteenth Century Journal Publishers, 1993, p. 175-204.

Frantz, David O., Festum Voluptatis. A Study of Renaissance Erotica, Columbus, Ohio State University Press, 1989.

Greene, Robert, The Life and Complete Works in Prose and Verse of Robert Greene. 15 vols, éd. Alexander B. Grosart, Londres, Huth Library, 1881-86, vol. 12.

Gurr, Andrew, « Professional Playing in London and Superior Cambridge Responses » in Shakespeare Studies, vol. XXXVII, éds. Susan Zimmerman et Garrett Sullivan, Madison, Fairlegh Dickinson University Press, p. 43-53.

Holderness, Graham, Nick Potter et John Turner, Shakespeare: Out of Court, Londres, Macmillan, p. 1990.

Jacquemier, Myriam, « Du désordre babélien à la conscience de l'altérité » in Ordre et désordre dans la civilisation de la Renaissance, Saint-Étienne, Publications de l'Université de Saint-Étienne, 1996, p. 123-38. 
Kibbee, Douglas A., For to Speke Frenche Trewely. The French language in England, 1000-1600. Its status, description and instruction, Amsterdam, John Benjamins Publishing Company, Studies in the History of the Language Sciences 60, 1991.

Lacan, Jacques, «Le séminaire livre XII, Les problèmes cruciaux pour la psychanalyse », 1964-1965 - 17 mars 1965. Non publié.

Marlowe, Christopher. Le Docteur Faust / Doctor Faustus, éd. François Laroque, traduction inédite par François Laroque et Jean-Pierre Villquin, Paris, GF Flammarion, 1997.

McPherson, David C. « Aretino and the Harvey-Nashe Quarrel », PMLA, Vol. 84, No. 6 (Oct., 1969): 1551-58.

Menon, Madhavi, «The L Words » in Shakesqueer: A Queer Companion to the Complete Works of Shakespeare, éd. Madhavi Menon, Durham, NC, Duke University Press, 2011, p. 187-94.

Nicholl, Charles, The Reckoning: The Murder of Christopher Marlowe, Chicago, The University of Chicago Press, 1992.

Northway, Kara, « Costard's Revenge: Letters and their Misdelivery in Love's Labour's Lost »in Renaissance Papers 2011, éds. Andrew Shifflett et Edward Gieskes, Camden House, New York, 2012, p. 17-30.

Palsgrave, John, L'éclaircissement de la langue française, Londres, 1530.

Puttenham, George, The Art of English Poesy (1589), éds. Frank Whigham et Wayne A. Rebhorn, Ithaca, Cornell University Press, 2007.

Rigolot, François, L'erreur de la Renaissance, Paris, Honoré Champion, 2002.

Sabot, Philippe, « Extase et transgression chez Georges Bataille ", Savoir et Clinique, 2007/1, n 8, p. 87-93. Site internet : http://www.cairn.info/zen.php?ID_ARTICLE=SC_008_0087 (consulté le 2 février 2015).

Shakespeare, William, Peines d'amour perdues, texte établi par Gisèle Venet, Traduction par JeanMichel Déprats, dans Comédies, tome 1, in CEuvres complètes, V, éds. Jean-Michel Déprats et Gisèle Venet, Paris, Gallimard, coll. « Bibliothèque de La Pléiade », 2013, p. 608-853.

Shakespeare, William, Love's Labour's Lost, éd. William C. Carroll, Cambridge, CUP, 2009.

Shakespeare, William, The Complete Works, éds. John Jowett, William Montgomery, Stanley Wells et Gary Taylor, $2^{\mathrm{e}}$ édition, Oxford, OUP, 2005.

Shell, Alison, Shakespeare and Religion, Londres, Methuen Drama, Bloomsbury, The Arden Shakespeare, 2010.

Whitney, Geffrey, A Choice of Emblemes, éd. John Manning, Aldershot, Scolar Press, 1989.

\section{NOTES}

1. Voir la définition du terme donnée par l'OED : «1a. Law. A wrongful act or omission; spec. a misdemeanour or failure of duty by a public official. [...] b. More generally: the mistaking of one thing for another; a misunderstanding; a mistake. »

2. Voir ce que dit Berowne au cours du dernier acte: "We to ourselves prove false / By being once false, for ever to be true ", V.ii.746-747. Les citations de Peines d'amour perdues sont tirées de l'édition de William C. Carroll (voir bibliographie). 
3. François Rigolot, L'erreur de la Renaissance, Paris, Honoré Champion, 2002, p. 205. Au chapitre VI («Erreurs et rhétorique ») de son ouvrage, Rigolot explique qu'Horace, dans l'Ars poetica, « avait osé parler des charmes des maculae ('fautes poétiques') qui sont le fruit d'une agréable incuria ('négligence'). »

4. Don Adriano de Armado, chevalier ab-errant, est le premier à prononcer ce mot apparemment tabou dans la comédie. Lorsque Holofernes décide confier le rôle d'Hercule à Moth, l'Espagnol s'exclame : « Pardon, sir, error! He is not quantity enough for the Worthy's thumb » (V.i.105-106). Il sera relayé par Berowne qui, venant d'être humilié par les Françaises au cours du bal des Moscovites, fait rimer « error » avec «terror » : « Now, to our perjury to add more terror, / We are again forsworn in will and error »(V.ii.470-471). Enfin, le même Berowne cherchera à se dédouaner partiellement de ses erreurs, commises au nom de l'amour, et en fera incomber partiellement la cause aux jeunes Françaises : « [...] Therefore, ladies, / Our love being yours, the error that love makes / Is likewise yours. [...]» (V.ii.744-746).

5. Il suffit en effet de se rappeler l'épitre dédicatoire de John Florio dans sa traduction des Essais de Montaigne, dédiée à six femmes de la famille Bedford-Harington : «French hath long time beene termed the language of Ladies: So doth it grace your tongues; so doe your tongues grace it; as if written by men it may have a good garbe, spoken by you it hath a double grace: for so I have heard some of you speake it, as no man, few women, could come near their sweete-relisht ayre of it ». Cité dans Juliet Fleming, « Dictionary English and the Female Tongue » in Privileging Gender in Early Modern England, éd. Jean R. Brink, Sixteenth Century Essays and Studies, vol. 23, Kirksville, MO, Sixteenth Century Journal Publishers, 1993, p. 188.

6. Ibid. Selon Juliet Fleming, "Florio's assertion that women make the best vernacular speakers had been inherited by the English humanists from Cicero ». Dans un passage extrait de Cicéron, De Oratore, Livre III, 44-46 (trad. H Rackham, Cambridge, Loeb Classical Library, 1982, p. 37), Crassus affirme en effet que les femmes préservent la pureté du Latin en le prononçant correctement.

7. Sur cette confusion répétée dans l'in-quarto puis dans l'in-folio, voir Oscar J. Campbell, «Love's Labour's Lost Restudied (1925) » in Love's Labour's Lost. Critical Essays, éd. Felicia Hardison Londré, New York, Routledge, 2001, p. 86 : «In the first quarto she is called 'queen' a number of times; and many of these designations are retained in the folio edition ». Campbell en conclut que cette désignation n'est pas une erreur et qu'on peut voir dans la Princesse un double dramatique de Marguerite de Valois qui, après s'être rendue à Nérac en 1578, trouva la cour de Navarre parfaitement à son goût.

8. William Shakespeare, Peines d'amour perdues dans Comédies, I, in CEuvres complètes, V, éds. JeanMichel Déprats et Gisèle Venet, Paris, Gallimard, 2013, p. 715.

9. Geffrey Whitney, A Choice of Emblemes, éd. John Manning, Aldershot, Scolar Press, 1989, p. 76 : «Heare Hanno standes, and looks into the skye, / And feedes him selfe, with hope of future praise: / Unto his birdes, he dothe his eare applie, / And trustes in tyme, that they his name should raise: / For they weare taught, before they flewe abrode, / Longe tyme to saie, that HANNO was a God. // But, when the birdes from bondage were releast, / And in the woodes, with other birdes weare join'de, / Then HANNOS name, theire wonted lesson ceaste, / For eache did singe, according to his kinde: / Then flee his faulte. Ambition works our shame, / And virtue love, which dothe extol our name ». L'emblème est également accessible sur le site Internet http:// www.mun.ca/alciato/wcomm.html (consulté le 18 mars 2015).

10. Voir la Bible, Genèse 1 : 26. Dans la King James Bible (1611), il est écrit : « And God said, Let us make man in our image, after our likeness: and let them have dominion over the fish of the sea, and over the fowl of the air, and over the cattle, and over all the earth, and over every creeping thing that creepeth upon the earth ». Voir le site http://www.kingjamesbibleonline.org/ Genesis-1-26/ (consulté le 18 mars 2015). 
11. Dans son essai de 1944, «Sur une philosophie de l'expression », paru dans "Poésie 44 » et portant sur les travaux de Brice Parain consacrés au langage, Albert Camus résumait ainsi l'idée profonde du philosophe. Albert Camus, Euvres complètes, tome I, éd. Jacqueline Lévi-Valensi, Gallimard, coll. La Pléiade, 2006, p. 908.

12. William Shakespeare, Peines d'amour perdues dans Comédies, I, in CEuvres complètes, V, ed. cit., p. 793.

13. Voir Alison Shell, Shakespeare and Religion, Londres, Methuen Drama, Bloomsbury, The Arden Shakespeare, 2010, p. 163.

14. L'attitude affectée d'Armado l'empêche par ailleurs d'être pris au sérieux par les autres : la gravitas propre à la cour lui fait défaut. Voir Graham Holderness, Nick Potter et John Turner, Shakespeare: Out of Court, Londres, Macmillan, 1990, p. 28.

15. Sur ce point, voir John Palsgrave, L'éclaircissement de la langue française, Londres, 1530, 214/1, «Disshecloute, souillon ». Voir aussi ce que dit la nourrice de Roméo dans William Shakespeare, Roméo et Juliette, III.v.219 : « Romeo's a dishclout to him (Paris) ».

16. Alors qu'il s'efforce de justifier la présence d'un page minuscule endossant le rôle d'Hercule, il croit sauver la face en mentionnant l'épisode où le héros, encore simple nourrisson, étrangle dans son berceau les serpents envoyés par Junon. Ce faisant, Holofernes ne suscite qu'une image scénique grotesque qui ne fait que souligner un peu plus l'incongruité de la distribution artistique de la pièce dans la pièce.

17. Comme l'a montré Antoine Compagnon, «[m]ultiplier les sententiae, recouvrir son discours d'yeux, de perspectives diverses et divergentes, c'est se défendre contre le regard de l'autre, mais c'est aussi s'exposer : faire de son discours un monstre, Argus qui surveille toutes les issues. 'Sed neque oculos esse toto corpore velim, ne caetera membra officium suum perdant'. Il ne faut pas que le discours soit couvert d'yeux, au risque que les autres membres de son corps soient mutilés : le corps merveilleux du discours doit être conforme aux canons de l'anatomie humaine, fidèle aux proportions du corps de l'orateur. » Antoine Compagnon, La seconde main ou le travail de la citation, Paris, Seuil, 1979, p. 146.

18. La version consultée dans le cadre de cet article est celle de 1603. Voir G. De la Mothe, The French alphabeth: teaching in a very short tyme, by a most easie way, to pronounce French naturally, to reade it perfectly, to write it truely, and to speake it accordingly. Together with The treasure of the French tongue, conteyning the rarest sentences, prouerbes, parables, similies, apothegmes and golden sayings of the most excellent French authours, as vvell poets as orators. The one diligently compiled, and the other painfully gathered and set in order, after the alphabeticall maner, for the benefite of those that are desirous of the French-tongue, Londres, 1603, STC n 6547.

19. Gilles (ou Georges) De la Mothe, actif en Angleterre à partir des années 1592, fut le tuteur du fils de Henry Wallop (Lord Chief Justice en Irlande). Il entra ensuite au service de la famille Wenman, dans l'oxfordshire, avant d'abandonner le tutorat en 1595 pour rejoindre les enseignants français qui officiaient alors à St Paul's Churchyard. Voir Douglas A. Kibbee, For to Speke Frenche Trewely. The French language in England, 1000-1600. Its status, description and instruction, Amsterdam, John Benjamins Publishing Company, Studies in the History of the Language Sciences 60, 1991, p. 193.

20. G. De la Mothe, The French alphabeth, ed. cit., p. $82:$ « The sweetnesse and facilitie of a toung consisteth in the multitude of vowels [...]. So that the toungs, wherein vowels exceede the nomber of consonantes, as the Greeke and Latin toung, and other their like, are much more sweeter to be pronounced, and pleasant to be heard, than those wherein for a vowel there is always three or foure consonants [...] That is the cause wherefore there be so many consonants in the French toung which are not pronounced [...]. »

21. Dans Gargantua, le précepteur Thubal Holoferne apprend à Gargantua à réciter l'alphabet à l'envers. 
22. George Puttenham, The Art of English Poesy (1589), éds. Frank Whigham et Wayne A. Rebhorn, Ithaca, Cornell University Press, 2007, p. 321 : «the Latines called it Congeries and we the heaping figure, as he that said To muse in minde how faire, how wise, how good, How brave, how free, how curteous and how true [...]. »

23. Randle Cotgrave, A Dictionarie of the French and English Tongues (Londres, 1611), facs., éd. William S. Woods, Columbia, The University of South Carolina Press, 1950. L'OED (1.a) définit comme suit ce mot, dont la première occurrence remonterait à Chaucer (c1398) : «a. The action of sending forth a poem; hence, the concluding part of a poetical or prose composition; the author's parting words; a dedication, postscript. Now chiefly the short stanza which concludes a poem written in certain archaic metrical forms. arch. ».

24. Se moquant de l'un des poèmes affectés de Gabriel Harvey, à savoir «Gorgon » (sous-titré "The Wonderful Year »), extrait de Supererogation (1593), Thomas Nashe en compare « Lenvoy » à un «fart after a good stool», ce qui en dit assez long sur la qualité littéraire du poème en question (ou sur la médisance de Nashe). On comprend dès lors que le clown associe en premier lieu « l'envoy » à l'administration annale d'un purgatif, avant d'entendre en lui le son « oi ». Voir à ce propos Charles Nicholl, The Reckoning: The Murder of Christopher Marlowe, Chicago, The University of Chicago Press, 1992, p. 60.

25. Myriam Jacquemier, « Du désordre babélien à la conscience de l'altérité » in Ordre et désordre dans la civilisation de la Renaissance, Saint-Étienne, Publications de l'Université de Saint-Étienne, 1996, p. 128, note 30 (123-138).

26. George Puttenham, The Art of English Poesy, ed. cit., p. 344.

27. Voici comment Derrida définit le supplément : « [...] le concept de supplément [...] abrite en lui deux significations dont la cohabitation est aussi étrange que nécessaire. Le supplément s'ajoute, il est un surplus, une plénitude enrichissant une autre plénitude, le comble de la présence. Il cumule et accumule la présence. C'est ainsi que l'art, la technè, l'image, la représentation, la convention, etc., viennent en supplément de la nature et sont riches de toute cette fonction de cumul. [...] Mais le supplément supplée. Il ne s'ajoute que pour remplacer. Il intervient ou s'insinue à-la-place-de; s'il comble, c'est comme on comble un vide. S'il représente et fait image, c'est par le défaut antérieur d'une présence. Suppléant et vicaire, le supplément est un adjoint, une instance subalterne qui tient-lieu. En tant que substitut, il ne s'ajoute pas simplement à la positivité d'une présence, il ne produit aucun relief, sa place est assignée dans la structure par la marque d'un vide. Quelque part, quelque chose ne peut se remplir de soi-même, ne peut s'accomplit qu'en se laissant combler par signe et procuration. » Jacques Derrida, De la grammatologie, II, 2, Paris, éditions de Minuit, 1967, p. 208.

28. Ibid.

29. Voir Madhavi Menon, «The L Words » in Shakesqueer: A Queer Companion to the Complete Works of Shakespeare, ed. Madhavi Menon, Durham, NC, Duke University Press, 2011, p. 190. Chez les hommes, majoritairement essentialistes, le signifié a mené au signifiant, tandis que les jeunes femmes, plutôt nominalistes, ont été capables de rompre le lien existant entre les deux.

30. Angel Day, The English Secretorie, Londres, imprimé par Robert Walde-graue, 1586, STC n 6401. 31. Andrew Gurr, "Professional Playing in London and Superior Cambridge Responses" in Shakespeare Studies, vol. XXXVII, éds. Susan Zimmerman et Garrett Sullivan, Madison, Fairlegh Dickinson University Press, p. 44.

32. Christopher Marlowe, Le Docteur Faust / Doctor Faustus, éd. François Laroque, traduction inédite par François Laroque et Jean-Pierre Villquin, Paris, GF Flammarion, 1997, p. 96, II.ii.89-90 : «Then Mephistopheles, receive this scroll, / A deed of gift of body and soul- ».

33. Georges Bataille, Histoire de l'œil (1928), Paris, Gallimard, 1993. Comme l'explique Philippe Sabot, « pour Bataille (...), l'extase naît (...) de la conversion du regard aux choses d'en bas, à ces mouvements les plus obscurs de l'existence qui, au lieu du simple dégoût ou de la honte, provoquent aussi bien le rire que l'angoisse. Or, l'œil représente et matérialise justement ce point 
de passage et d'inversion du haut et du bas, de l'honnête et de l'obscène ». Philippe Sabot, «Extase et transgression chez Georges Bataille», Savoir et Clinique, 2007/1, n 8, p. 87-93, paragraphe 13. Voir le site : http://www.cairn.info/zen.php?ID_ARTICLE=SC_008_0087 (consulté le 2 février 2015).

34. Un exemple suffira à illustrer nos propos. Boyet, voulant prendre congé, s'exclame : «I fear too much rubbing. Good night, my good owl » (IV.i.132). À l'issue d'une scène qui donne lieu à de multiples sous-entendus sur les bêtes à cornes à et maints jeux de mots grivois, on est en droit de se demander si « rubbing " ne se rapporte vraiment qu'au jeu de boules mentionné par le clown, (qui pense que Maria, trop forte au tir à l'arc, pourrait être vaincue sur un autre terrain), ou si le mot n'évoque pas plutôt la masturbation. Dans un tel contexte, le mot «owl » pourrait bien renvoyer à « hole ", au sexe féminin.

35. Randle Cotgrave, A Dictionarie of the French and English Tongues, ed. cit.

36. Voir à ce sujet Kara Northway, "Costard's Revenge: Letters and their Misdelivery in Love's Labour's Lost » in Renaissance Papers 2011, éds. Andrew Shifflett et Edward Gieskes, Camden House, New York, 2012, p. 17-30.

37. Pour mémoire, Robert Greene s'adresse à Thomas Nashe en le qualifiant de "young Juvenall » dans Greene's Groatsworth of Wit (1592). Voir Robert Greene, The Life and Complete Works in Prose and Verse of Robert Greene. 15 vols. Ed. Alexander B. Grosart, Londres, Huth Library, 1881-86, vol. 12, p. 143.

38. Sur Nashe perçu comme un «self-styled English Aretino", voir David O. Frantz, Festum Voluptatis. A Study of Renaissance Erotica, Columbus, Ohio State University Press, 1989, p. 188. Voir aussi David C. McPherson, « Aretino and the Harvey-Nashe Quarrel », PMLA, Vol. 84, No. 6 (Oct., 1969) : 1551-58.

39. Natale Conti, Mythologie c'est à dire, Explication des Fables contenant les genealogies, Lyon, 1600, « De Cupidon », p. 397.

40. Ibid., p. 394.

41. Au cours de l'acte 3, alors que Costard vient d'obtenir une « rémunération » d'Armado qui lui a confié sa lettre pour Jaquenetta, il décrit déjà son bienfaiteur comme «My sweete ounce of mans flesh, my [in-conie] Jew ! » (3.1.118).

42. Une ressemblance qu'il exploite aussi dans sa traduction des Élégies d'Ovide (I.x). Selon David Crystal, « [incony] probably related to cony, 'rabbit', which developed as a term of male-to-female endearment. It was pronounced 'cunny', rhyming with money and honey, and this pronunciation inevitably gave it an indecent association, which was also current around 1600 ». Voir David Crystal, 'Think on my Words'. Exploring Shakespeare's Language, Cambridge, CUP, 2008, p. 169.

43. Chez Shakespeare, le mot " wit ", dérivé du français « vit », peut en effet désigner les organes sexuels masculin et féminin. Voir par exemple William Shakespeare, As You Like It, éd. Alan Brissenden, Oxford, OUP, 1998, Appendix A, p. 231.

44. « We did not quote them so ", rétorque ainsi Rosaline à Longaville (V.ii.760).

45. William Shakespeare, Peines d'amour perdues dans Comédies, I, in CEuvres complètes, V, ed. cit., p. 626.

46. À l'origine, il s'agit de la devise de Frédéric III signifiant «Le monde entier sera sous le commandement de l'Autriche ». Voir Benjamin Curtis, The Habsburgs: The History of a Dynasty, London, Bloomsbury, 2013, p. 1.

47. François Rigolot, L'erreur de la Renaissance, ed. cit., p. 364.

48. Jacques Lacan, "Le séminaire livre XII, Les problèmes cruciaux pour la psychanalyse ", 1964-1965 - 17 mars 1965. Non publié. 


\section{RÉSUMÉS}

Comédie de la copia et de l'ignorance, Peines d'amour perdues est une œuvre riche en approximations, faisant ainsi la part belle à l'erreur, même si le mot lui-même n'y est que fort peu employé, n'apparaissant paradoxalement que dans le dernier acte qui devrait être celui de la résolution plutôt que de la confusion. Au cœur d'une Navarre shakespearienne rongée par l'illusion et l'incertitude, l'erreur renvoie au péché originel et à ce serio ludere si prisé par les humanistes de la Renaissance. Ainsi, le dramaturge n'a de cesse de transformer fautes, faiblesses et fourvoiements en créations langagières mettant l'erreur à l'honneur pour mieux la transfigurer sur le plan dramatique. Mais, derrière pareille virtuosité et une apparente légèreté, l'erreur, dans la pièce, ne servirait-elle pas aussi à mettre en avant les failles et les fragilités de l'humanité ? Cet article s'interrogera donc sur le véritable statut de l'erreur dans cette comédie discordante et sur la manière dont Shakespeare, animé par un scepticisme aussi profond que joyeux, s'amuse à en subvertir puis à en réécrire la ou les définition(s).

As a comedy devoted to copia and ignorance, Love's Labour's Lost is full of shortcomings and approximations. It thus sheds light on its numerous errors, even though the word "error" seldom crops up in the text and is only mentioned in the last act of the play - something of a paradox, since act $\mathrm{V}$ normally provides a form of resolution rather than confusion. At the heart of a Shakespearean Navarre dominated by illusion and uncertainty, error is linked to the original sin and to the serio ludere tradition so much praised by the Renaissance Humanists. The playwright turns weaknesses, errors and misprision into linguistic creations that serve to enhance error through some sort of dramatic transfiguration. Yet, beyond the play's verbal virtuosity and apparent lightness, one can wonder whether all the errors in the play do not also serve to emphasize the defects and weaknesses of mankind. This article will thus analyse the real status of error in Shakespeare's jarring comedy by examining how the playwright, in a deep but merry form of scepticism, joyfully subverts and rewrites the usual definition(s) of error.

\section{INDEX}

Mots-clés : erreur, ignorance, excès, transgression, chaos, felix culpa, periergia, scepticisme

Keywords : error, ignorance, excess, transgression, chaos, felix culpa, periergia, scepticism

\section{AUTEUR}

\section{SOPHIE CHIARI}

Université Blaise Pascal, Clermont-Ferrand, CERHAC, UMR 5037 du CNRS 